\title{
Practical bioinstrumentation developments for AC magnetic field- mediated magnetic nanoparticle heating applications
}

\author{
Mahendran Subramanian $^{1}$ (D) Arkadiusz Miaskowski ${ }^{2} \cdot$ Ajit K. Mahapatro $^{3} \cdot$ Jon Dobson $^{4}$
}

Received: 13 November 2018 / Accepted: 7 February 2019 / Published online: 19 February 2019

(c) The Author(s) 2019

\begin{abstract}
Heat dissipation during magnetization reversal processes in magnetic nanoparticles (MNP), upon exposure to alternating magnetic fields (AMF), has been extensively studied in relation to applications in magnetic fluid hyperthermia (MFH). This current paper demonstrates the design, fabrication, and evaluation of three innovative advances in bioinstrumentation research, operating on this principle, for use as (i) a non-contact, in vitro, real-time temperature monitor; (ii) a drug release analysis system (DRAS); and (iii) a high flux density module for AMF-mediated MNP studies. The proposed DRAS is demonstrated by an AMF-mediated drug-release proof-of-principle experiment. Also, the technique described facilitates non-contact temperature measurements of specific absorption rate (SAR) as accurately as temperature measurements using a probe in contact with the sample. Numerical calculations estimating the absolute and root mean squared flux densities, and other MNP-AMF studies suggest that the proposed stacked planar coil module could be employed for calorimetry.
\end{abstract}

\section{Introduction}

The current aspiration of magnetically targeted/triggered drug and biomolecule delivery is to minimize the quantities of drugs used in therapies and to more effectively deliver them to the therapeutic target. The demands of modern instrumentation mean that high-precision tools are needed to overcome issues when developing and standardizing experimental methodologies [1]. This influences the ability of future technologies to meet the demand for the accurate detection of electrical, magnetic, optical, and thermal signals using sensitive, high-precision tools. In the past decade,

Mahendran Subramanian

m.subramanian@imperial.ac.uk

1 Departments of Bioengineering and Computing, Royal School of Mines, Imperial College London, London SW7 2AZ, UK

2 Department of Applied Mathematics and Computer Science, University of Life Sciences Lublin, Gleboka 29, Lublin, Poland

3 Department of Physics and Astrophysics, University of Delhi, Delhi 110007, India

4 Department of Material Science and Engineering, J. Crayton Pruitt Family Department of Biomedical Engineering, and Institute for Cell and Tissue Science and Engineering, ICTSE, University of Florida, PO Box 116131, Gainesville, FL 32611, USA alternating magnetic field (AMF) mediated magnetic nanoparticle (MNP) heating has attracted a great deal of attention due to its potential in biomedical applications.

Non-contact, in vitro temperature measurement techniques, such as infrared thermography and IR sensors, has been difficult due to the requirement for direct optical exposure to the sample. Scientists and engineers from multidisciplinary research areas have developed instruments which use very specific algorithms [2]. These needs to be standardized so that well-evaluated and easy to use tools, compatible with biomedical applications, can be designed. With the currently available instruments, the temperature sensing used for the magnetic fluid calorimetry is recorded by utilizing a thermocouple, an infrared sensor, infrared thermography, or fibre-optic sensors [3]. However, each of these techniques has its own advantages and disadvantages. Thermocouples are made up of metals (copper and constantine) and when exposed to AMF, metals generate heat due to Eddy currents, produce radio-frequency (RF) interference, and develop corrosion after repeated use when they are immersed within the colloid. Contamination of samples is also possible when using the same thermocouple for repeated calorimetric measurements. This means that metal thermocouple probes are not really usable in the presence of AMF [4-6]. Infrared thermography and infrared sensor modules require direct optical exposure to the in vitro sample [3,7]. This involves heat loss from the sample and carries the risk of sample 
contamination. Similarly, a fibre-optic sensor is not prone to electromagnetic interference but, as mentioned above, does have to have direct contact with the sample. In addition, these are expensive, delicate (silicone tube), and the probability of cross contamination is high. This is one of the reasons for the limited number of experiments using real-time temperature measurements in magnetic fluid hyperthermia research involving cell cultures; such experiments require a contamination-free environment [8-10]. MRI mediated noncontact temperature measurement is plausible, but, customisation, accessibility, ease of use and cost involved require consideration [11].

AMF-mediated drug delivery is popular because of the fact that it is non-invasive and remotely triggered. Controlled drug delivery systems have been a broad research interest for a number of years. The stimuli used here is the heat dissipated from surface functionalised magnetic nanoparticles exposed to AMF [12-14]. There are a few crude setups for performing such experiments using commercially available AMF systems [15, 16], and the research community has discussed the prospects for the development of nanoparticlebased drug delivery testing apparatus [17]. However, this requires certain modifications to be made to the United States Pharmacopeia (USP) type drug testing setups generally used in the pharmaceutical industry. Such modifications would have to take the following into account: nanoscale level fillers/binders, release patterns, the stimuli involved, the AMF generator, stirring mediated convection loss, precise sample positioning, the requirement for a water jacket, and the sampling method. The instrument development considered here looks at USP and flow-cell type apparatus in relation to the designing and fabrication of AMF-mediated drug delivery testing setups which facilitate precise physical and chemical measurements.

Biomedical application research within this topic has advanced significantly over the past decade; however, robust and well-evaluated tools that will allow researchers to replicate experiments are a must. Herein, we provide insights into development of practical modules for commercialization. This paper presents our recent innovative advances in bioinstrumentation research and demonstrates three different elementary but effective tools that could benefit researchers working in fields related to AMF-mediated MNP heating. These include; (1) non-contact in vitro temperature measurement with an 8 turn single layer planar coil setup; (2) drug-release analysis setup utilizing a solenoid coil; and (3) the utilization of stacked planar coils with increased flux density at low current input for calorimetric measurements.

Preliminary testing of our non-contact temperature measuring technique for the evaluation of specific absorption rate (SAR) estimated $8.88 \pm 0.24 \mathrm{~W} / \mathrm{g}$ for a test MNP containing $10 \mathrm{mg} / \mathrm{ml}$ aqueous suspension of dimercaptosuccinic acid stabilized magnetic nanoparticles. These results were in good agreement with the value of $8.83 \pm 0.15 \mathrm{~W} / \mathrm{g}$ as yielded by a temperature measurement probe which was placed in contact with the MNP. Likewise, we have designed, fabricated, and evaluated an AMF-mediated drug-release testing setup; a 2-layer stack of planar coils that provides increased flux density for calorimetric experiments (with no harmonics at low current input). This module generates high flux density with low coil current, uses high frequencies, and provides uniform and homogeneous incident flux density to expose onto the calorimetric sample. This stacked planar coil system was evaluated, in combination with an associated high voltage variable capacitance module, for its ability to produce electromagnetic frequencies between 50 and $250 \mathrm{kHz}$.

\section{Materials and methods}

CAD was used to design the systems and components were fabricated using precision engineering and $3 \mathrm{D}$ printing. Physical measurements of MNP heating under RF exposure and numerical model based calculations were performed to evaluate the effectiveness of the proposed techniques and design.

\subsection{AMF generating device}

We used magneTherm ${ }^{\mathrm{TM}}$ (nanotherics Ltd, Staffordshire, UK) system for generating AMF. The standard system encloses 17 turns, and a 9 turns solenoid coils that are capable of generating AMF up to $25 \mathrm{mT}$ within the range of $100 \mathrm{kHz}-1 \mathrm{MHz}$. The setups described in this article were designed to either fit within the magneTherm ${ }^{\mathrm{TM}}$ system or replace the standard solenoid coil modules.

\subsection{Non-contact temperature measurement}

The Live cell-Alternating Magnetic Field ${ }^{\mathrm{TM}}$ (LC-AMFTM) module connected to a magneTherm ${ }^{\mathrm{TM}}$ system enables RF exposure to an MNP sample in a petri dish (Lumox ${ }^{\circledR} 35 \mathrm{~mm}$, Sarstedt, Numbrecht, Germany), with or without cells [10]. LC-AMFTM setup encloses an 8 turn single layer planar coil. An ultra-thin polystyrene (or other polymer) film in a cellculture petri dish is formed as a base, and used as a window for gas exchange and non-contact temperature measurements. The fibre-optic temperature sensor (OTG-M360-1062ST-1.5PTFE-XN-10GT-M2; OPSENS, Quebec, Canada) measurements were undertaken by attaching the sensor tip to the bottom of the petri dish; this allowed the recording of the temperature of the underside of the film. In the case of the mid-infrared temperature sensor (CSmicro 2W Mid-Infrared (MIR), Optris, Berlin, Germany), with a working range of 
8-14 $\mu \mathrm{m}$, the sensor head was situated on the underside of the film.

\subsection{Computer aided design (CAD) and computer aided Machinery}

Drug-release analysis system and coil forms were designed using CAD. All CAD work was done using PTC Creo v3.0 (Staffordshire, UK) and the 3D printing was depicted using Flashforge-Creator pro (London, UK).

\subsection{Stacked planar coil fabrication}

Hollow water-cooled coils were fabricated using 18 standard wire gauge (SWG) copper wire (Maplin, Stoke on Trent, UK) and silicon tube (Saint-gobain, Coventry, UK) with dimensions $4 \mathrm{~mm}$ ID $\times 6 \mathrm{~mm}$ OD. A coil form (bobbin) was designed and precision engineered for use in winding and fixing the geometry of the coil.

\subsection{Microprocessor programming}

The interfacing software was programmed in $\mathrm{C}++$ on an off-line development system. The software was run on an Arduino Uno microprocessor board driving a motor shield v1.0 (Farnell, Angus, UK). The Arduino IDE platform was used to upload the firmware onto the microprocessor.

\subsubsection{Experimental and numerical evaluation}

2.5.1.1 Calorimetric experiment $20 \mathrm{mg} / \mathrm{ml}$ aqueous suspensions of $50 \mathrm{~nm}$ sized maghemite (Sigma Aldrich, Dorset, UK) and $10 \mathrm{mg} / \mathrm{ml}$ aqueous suspensions of dimercaptosuccinic acid (DMSA) stabilized $10.3 \mathrm{~nm}$ sized magnetite (Hypermag A, nanoTherics, Staffordshire, UK) were used for the calorimetric and drug-release testing measurements. Identical containers were used in all the calorimetric experiments. A Lumox ${ }^{\circledR} 35 \mathrm{~mm}$ petri dish (Sarstedt, Numbrecht, Germany) was used for the in vitro temperature measurements and a $3 \mathrm{ml}$ Pur-A-Lyser dialysis tube (Sigma Aldrich, Dorset, UK) was used for testing the drug release. MNPs were subjected to vortex and ultra-sonicate prior to AMF exposure. A Pico $\mathrm{M}^{\mathrm{TM}}$ with OTG-MPK 5 optical sensor system (Opsens, Quebec, Canada) and an Osensa fibre-optic temperature sensor (Burnaby, Canada) were used for recording real-time temperature measurements. An alternating current (AC) magnetic field-magneTherm system (nanoTherics, Staffordshire, UK) was used as a source of exposure. The specific loss power (W/g of particles) was calculated using the following equation:

$\mathrm{SAR}=\left(\frac{C}{\phi}\right)\left(\frac{\Delta T}{\Delta t}\right)$ where $C$ is the specific heat capacity of the MNP in $\mathrm{J} /$ $\mathrm{Kml}, \phi$ is the concentration of MNP in $\mathrm{mg} / \mathrm{mL}$, and $\Delta T / \Delta t$ is the rate of change of temperature over time. An appropriate region of the graph was utilized, using the corrected slope method, for calculations [18]. The baseline temperature was subtracted from the results, and the linear loss parameter was estimated from the temperature difference over time slopebased on fitting interval and number of fits.

2.5.1.2 Spectroscopy measurements Absorption spectroscopy was performed using a Shimadzu UV-Vis spectrophotometer (Milton Keynes, UK); the samples were placed in a quartz crystal cell. Real-time UV-Vis absorption spectroscopy data was recorded using a non-metal fibre-optic probe (FCB-UV200-1.5-S1, Avantes, Surrey, UK) connected to a halogen light source (Visual and near infrared range, Avantes, Surrey, UK) and a spectrophotometer module (Visual range, Avantes, Surrey, UK) while performing the AMF-mediated drug-release experiment. The cancer drug-doxorubicin hydrochloride-was procured from Sigma Aldrich (Dorset, UK).

2.5.1.3 Flux density simulation and calculation The magnetic flux density $(B)$ was simulated. This was done by the Sim4Life (ZMT Zurich MedTech, Zurich) platform. The flux distribution was modelled in relation to an applied low frequency magneto-quasi static (LF M-QS) algorithm utilizing the finite element method (FEM) model. Here, the coil wire was treated as a perfect electric conductor (PEC) and the parameters defining the coil geometry used in the numerical modelling included: the outer diameter of the tube $\left(D_{t}\right)$, the inner diameter of the tube $\left(d_{t}\right)$, the use of 18 standard wire gauge copper wire $\left(g_{\mathrm{cu}}\right)$, the number of turns $(\mathrm{N})$, the number of layers $(l)$, the distance between the turns $\left(S_{\mathrm{N}}\right)$, the distance between layers $\left(S_{1}\right)$, the inner diameter of the coil $\left(d_{\mathrm{c}}\right)$, and the outer diameter of the coil $\left(D_{\mathrm{c}}\right)$. Two coils were modeled, i.e., the double stack planer one and the curved planar one.

For the double-stacked planar coil with 14 turns each the simulation with $D_{\mathrm{t}}=6 \mathrm{~mm}, d_{\mathrm{t}}=4 \mathrm{~mm}, g_{\mathrm{cu}}=$ $1.22 \mathrm{~mm}, N=14,1=2, S_{\mathrm{N}}=3.208 \mathrm{~mm}, S_{1}=6.78 \mathrm{~mm}, d_{\mathrm{c}}=$ $17.208 \mathrm{~mm}$, and $D_{\mathrm{c}}=132.336 \mathrm{~mm}$.

\section{Results and discussion}

\subsection{Non-contact temperature monitor}

A schematic can be found in Fig. 1b to understand positioning of the probes in this section dedicated to non-contact temperature measurement. Figure 2a plots the temperature variation within the exposure time of a magnetic field of strength $5 \mathrm{mT}$ at an AC frequency of $218.7 \mathrm{kHz}$, recorded 

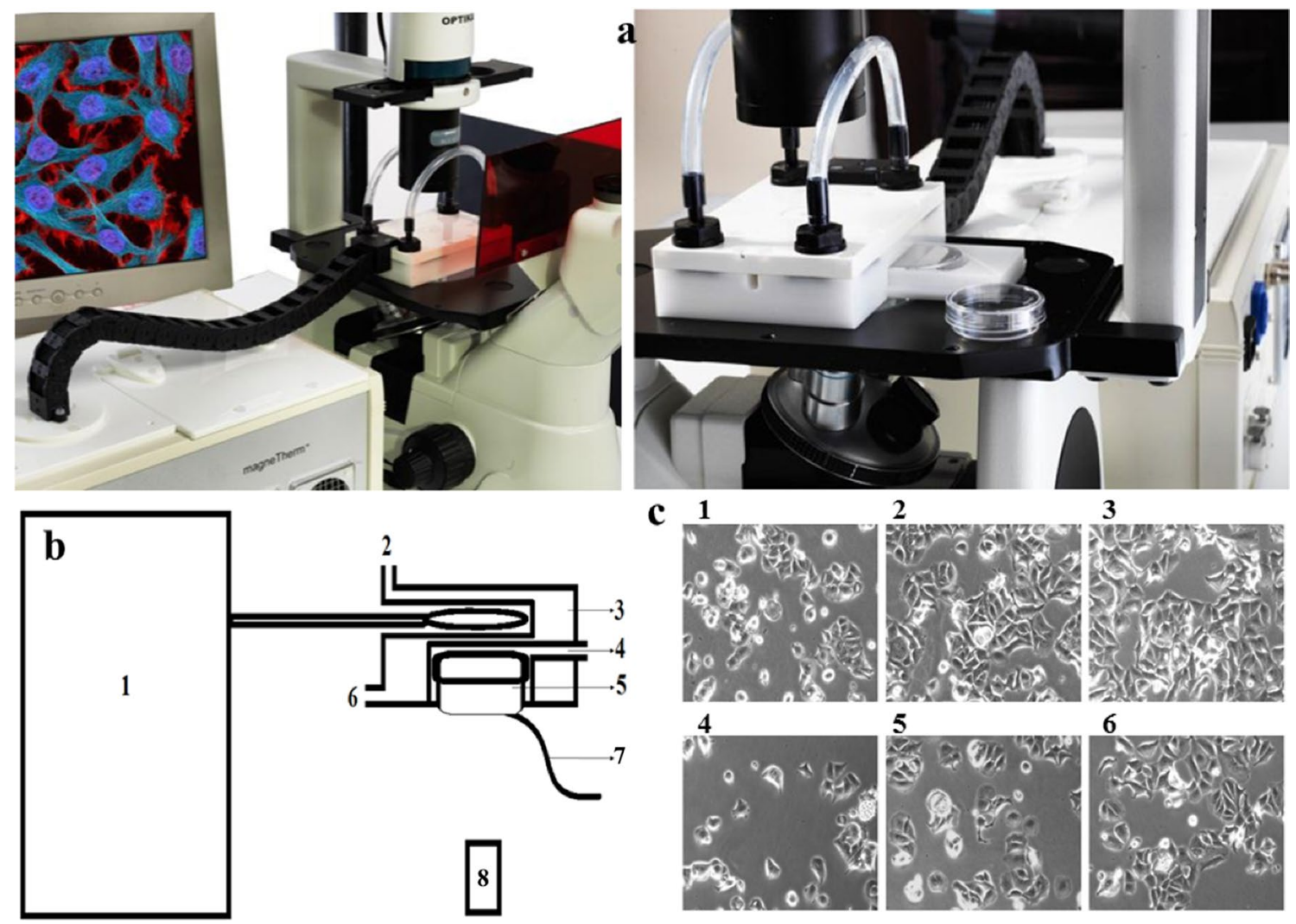

Fig. 1 a Non-contact temperature measurement setup-the coil system (enclosing a single layer 8 turn planar coil) used for realtime magnetic fluid hyperthermia microscopy studies. b Block diagram schematic of the proposed technique and the apparatus used. b1-alternating magnetic field applicator, which can generate timevarying magnetic fields of $1 \mathrm{kHz}-1.2 \mathrm{MHz}$ and $20 \mathrm{mT}$ flux density; b2 - cell-culture-incubation chamber's water inlet; b3-cell-culture incubation chamber's water jacket to maintain physiological temperature; b4-biological gas flow; b5—cell-culture vessel with $50 \mu \mathrm{m}$

or less base thickness; further referred to as a $35 \mathrm{~mm} 2$ cell-culturetreated petri dish. b6 - cell-culture-incubation chamber's water outlet; b7-fibre-optic temperature sensor; b8-infrared temperature sensor; further referred to as CSmicro 2W Mid-Infrared (MIR) 8-14 $\mu \mathrm{m}$ temperature sensor (Optris, Berlin, Germany). c MCF 7 breast cancer cells were seeded on day 0 and maintained within the setup shown in (a) up to day 3; c1, 2, 3- day 1-3 cells grown within the coil setup incubator; c4, 5, 6-day 1-3 control cells grown in laboratory cell incubator

simultaneously using a fibre-optic temperature sensor in contact with the radio-frequency susceptible nanoparticles and an infrared temperature sensor connected through the base of a petri dish. Very small differences in the measurements of the absolute temperatures using these two probes indicate that the mid-infrared region can measure the temperature of the MNP sample through/ on the permeable material during an AMF exposure. The comparison between the two fibre-optic temperature sensors used for recording the temperature variation in the MNPs through the exposure time of the magnetic field is plotted in Fig. $2 b$.

Here, one sensing probe was directly in contact with the radio-frequency susceptible MNP, and the other was not in direct contact, but measured the temperature through the base of the petri dish. The temperatures measured by both the in contact and the non-contact fibre-optic probes were used to calculate SAR values for the MNP sample-by

considering the entire region of the change of temperature over the entire AMF exposure time using the corrected slope method [18]; this is represented in Fig. 2c.

The value of SAR which was based on the non-contact temperature measurement, $\mathrm{SAR}=8.88 \pm 0.24 \mathrm{~W} / \mathrm{g}$, agreed well with the value of $8.83 \pm 0.15 \mathrm{~W} / \mathrm{g}$, as calculated using the temperature measurement probe which was in contact with the MNP sample. The method being developed here, demonstrated its ability to accurately perform non-contact temperature measurement. It is a suitably cost-effective method for the non-contact monitoring of in vitro samples and could make redundant the complex algorithms that have been proposed [19] to enable the use of non-contact temperature measurement via mid-infrared pyrometersthese cannot measure temperature through the commonly used polypropylene sample tubes and glass petri dishes. The advantage of this type of setup is the ability to perform 

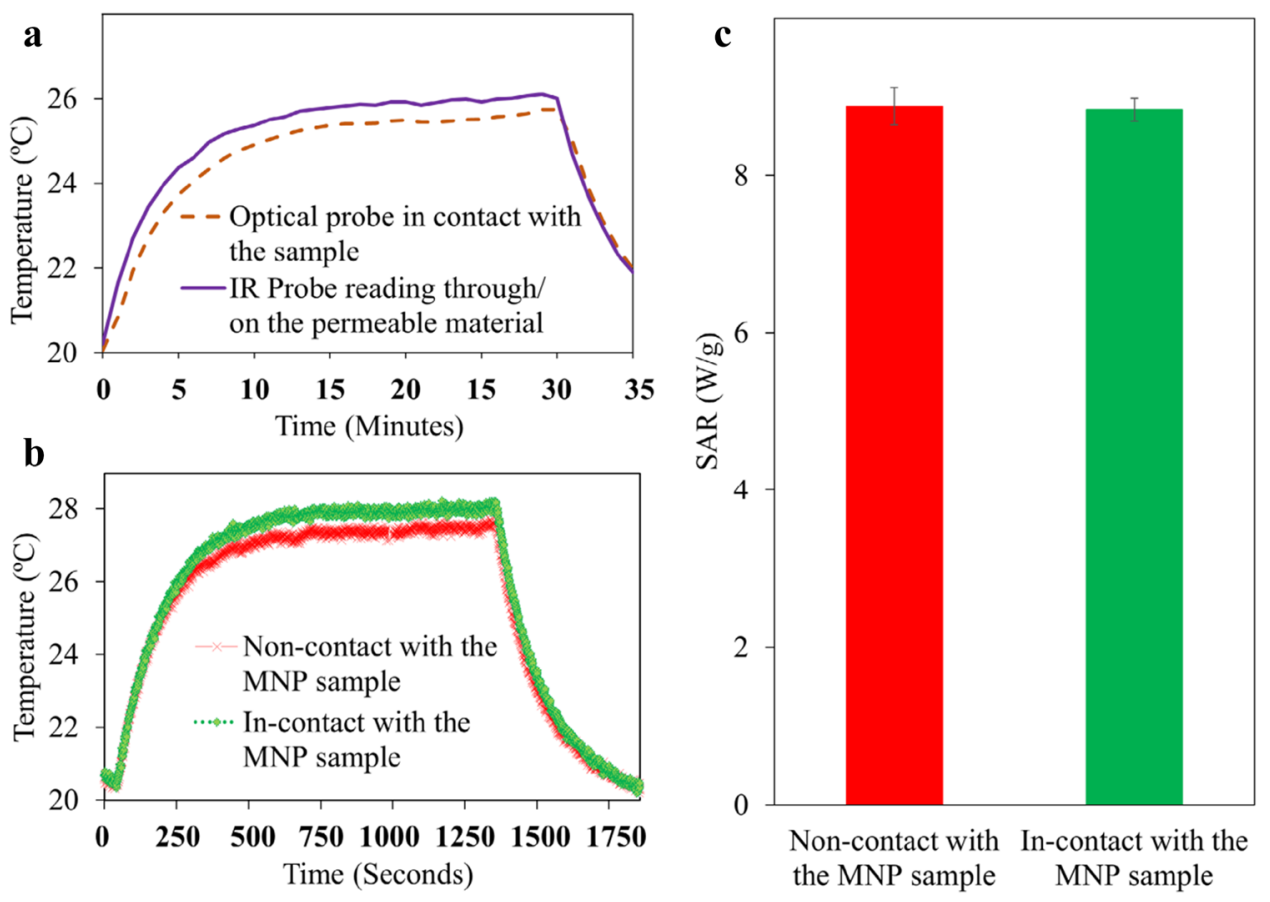

Fig. 2 a Temperature variation through the exposure time of a magnetic field with strength $5 \mathrm{mT}$ at an AC frequency of $218.7 \mathrm{kHz}$, recorded simultaneously using a fibre-optic temperature sensor (OTG-M360-10-62ST-1.5PTFE-XN-10GT-M2; OPSENS, Quebec, Canada) in contact with the radio-frequency susceptible nanoparticles and an infrared temperature sensor (CSmicro 2W Mid-Infrared (MIR), Optris, Berlin, Germany) with a working range of 8-14 $\mu \mathrm{m}$, connected through the base of the petri dish. b A comparison between two fibre-optic temperature sensors (OTG-M360-10-62ST-1.5PTFEXN-10GT-M2, OPSENS, Quebec, Canada) used for recording the temperature variation throughout the exposure time of the magnetic field. One sensing probe was placed in contact with the radio-fre-

non-contact temperature measurements using a fibre-optic sensor, an infrared pyrometer and an infrared camera. However, the petri dish itself is a limitation of this experimental setup as different types of MNP samples have varying heat dissipation profiles and the thin polystyrene (or other polymer) layer- $70{ }^{\circ} \mathrm{C}$ will have a temperature-resistant tolerance. Furthermore, near infrared wavelength window of the pyrometer deployed here only records surface temperature.

\subsection{Drug-release system}

The whole setup was designed using CAD and fabricated to fit within the solenoid coil setup as commercially available components did not fit the required specifications. Figure $3 \mathrm{a}$ highlights the unique features of the proposed drug-release testing setup including: (i) a disposable dialysis tube holding a shaft capable of accommodating a $250 \mu \mathrm{L}-3 \mathrm{~mL}$ volume of sample; (ii) a high resolution fibre-optic temperature sensor for real-time temperature measurements; (iii) the sampleholding shaft driven by a stepper motor to allow continuous quency susceptible MNP, and the other measured the temperature through the base of the petri dish without any direct contact with the radio-frequency susceptible nanoparticle sample. c Specific absorption rate (SAR) values calculated from the changes in temperature with time, using both of the fibre-optic temperature sensors (two OTG-M360-10-62ST-1.5PTFE-XN-10GT-M2; OPSENS, Quebec, Canada), one in contact with the radio-frequency susceptible nanoparticle sample and the other connected to the base of the petri dish. There is no significant difference $(\% 0.5)$ between the SAR values calculated using the temperatures yielded by the probe in contact and the probe not directly in contact with the sample. DD $\mathrm{H}_{2} \mathrm{O}$ only and MNP only with and without AMF exposure were used as controls

stirring of the solution - for enabling even distribution of the drug released into the dissolute; (iv) the sampling port/ shaft for manual spectrophotometer analysis and to accommodate a custom-engineered non-metal fibre-optic probe for real-time UV-Vis spectrophotometer analysis, while performing the AMF-mediated drug-release experiment; and (v) a water jacket enclosing the dissolute holding chamber to maintain ambient temperature in the dissolute as well as in the MNP + Drug sample. The outer layer of the water jacket is a dielectric shield. These features are appropriate for the design of a working prototype (as shown in Fig. 3b-d) and for recording SAR measurements at high precision.

Here, the steps produced by the stepper motor are critical, as an increased rotation per minute (rpm) rate might increase heat dissipation by the sample into the surrounding dissolute. Additionally, the volume of dissolute is critical as the stimulus involved for drug release is heat. Generally, $20 \mathrm{rpm}$ and $25 \mathrm{ml}$ of dissolute volume were used in this study. This dissolute volume was used so that the dialysis tube stayed immersed [16], and is 12.5 times more than the 

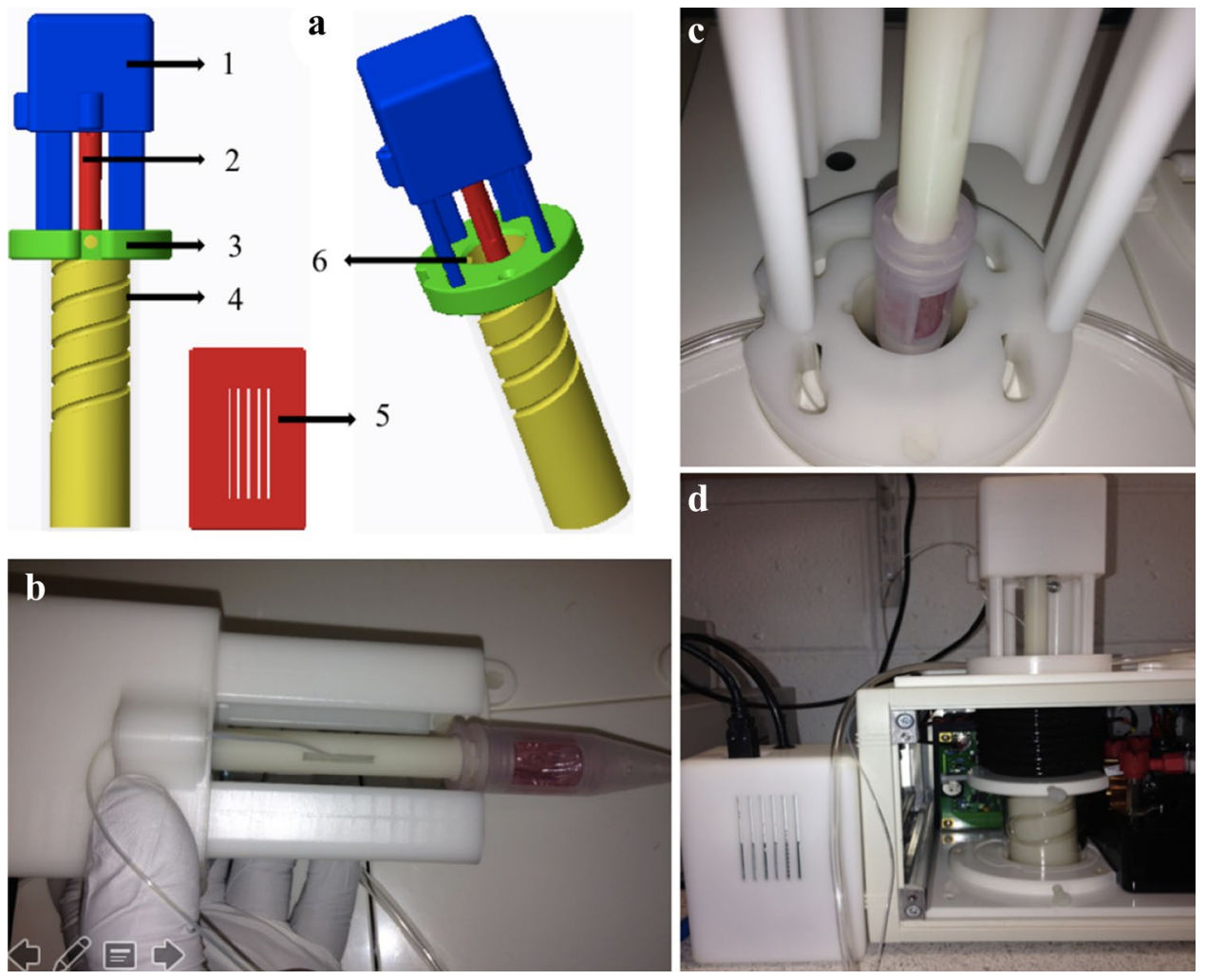

Fig. 3 Various parts of the AMF-mediated drug-release testing setup (a): (a1) the stepper motor mounting; (a2) the dialysis tube sealing shaft with an aperture for a temperature probe attached to the stepper motor, (a3) a magneTherm ${ }^{\mathrm{TM}}$ mounting clamp; (a4) the water jacket (bottom sealed); (a5) the control box; and (a6) the sampling port. b A photograph of the AMF-mediated drug-release analysis setup the stepper motor attached to the $12 \mathrm{kDa}$ dialysis tube through a shaft - the shaft also provides temperature probe access to the MNP sample; $\mathbf{c}$ the dialysis tube attached to the stepper motor positioned within the dissolute; $\mathbf{d}$ and the complete setup with the water jacket attached to the magneTherm ${ }^{\mathrm{TM}}$ coil enclosure including the microprocessor (within control box), programmed to provide oscillating motion. d Shows the final DRAS setup - the dielectric shield enclosing the water jacket is visible (below the solenoid coil). The double layer 17 turns-water-cooled solenoid coil (black colour) is visible. The DRAS setup is designed to fit within the solenoid coil (Internal diameter-50 $\mathrm{mm}$ )

drug-release testing setup. The rotation can be adjusted from 120 to 20 RPM to mimic in vivo blood flow. The design suitability was further enhanced by a water jacket fabricated in clear material to enable visualization of the released drug into the medium. Displacement spacers were added to the water jacket design to perform experiments with different volumes of drug-release medium.

The steps produced by the stepper motor and volume of dissolute are critical factors. Variation of volume of dissolute does influence the heat dissipation from the MNP + Drug conjugate. Moreover, the heat dissipation from the MNP: Volume of dissolute ratio depends upon the magnetic and chemical properties of the MNP + Drug conjugate and the flux parameters of the magnetic field applicator. Herein, aqueous suspension of commercially available $50 \mathrm{~nm}$ sized maghemite particles were conjugated with Doxorubicin and exposed to AMF to demonstrate the function of the proposed design in vitro. The change of temperature curve shows that heating ranges from 23 to $67^{\circ} \mathrm{C}$ after AMF exposure for this 

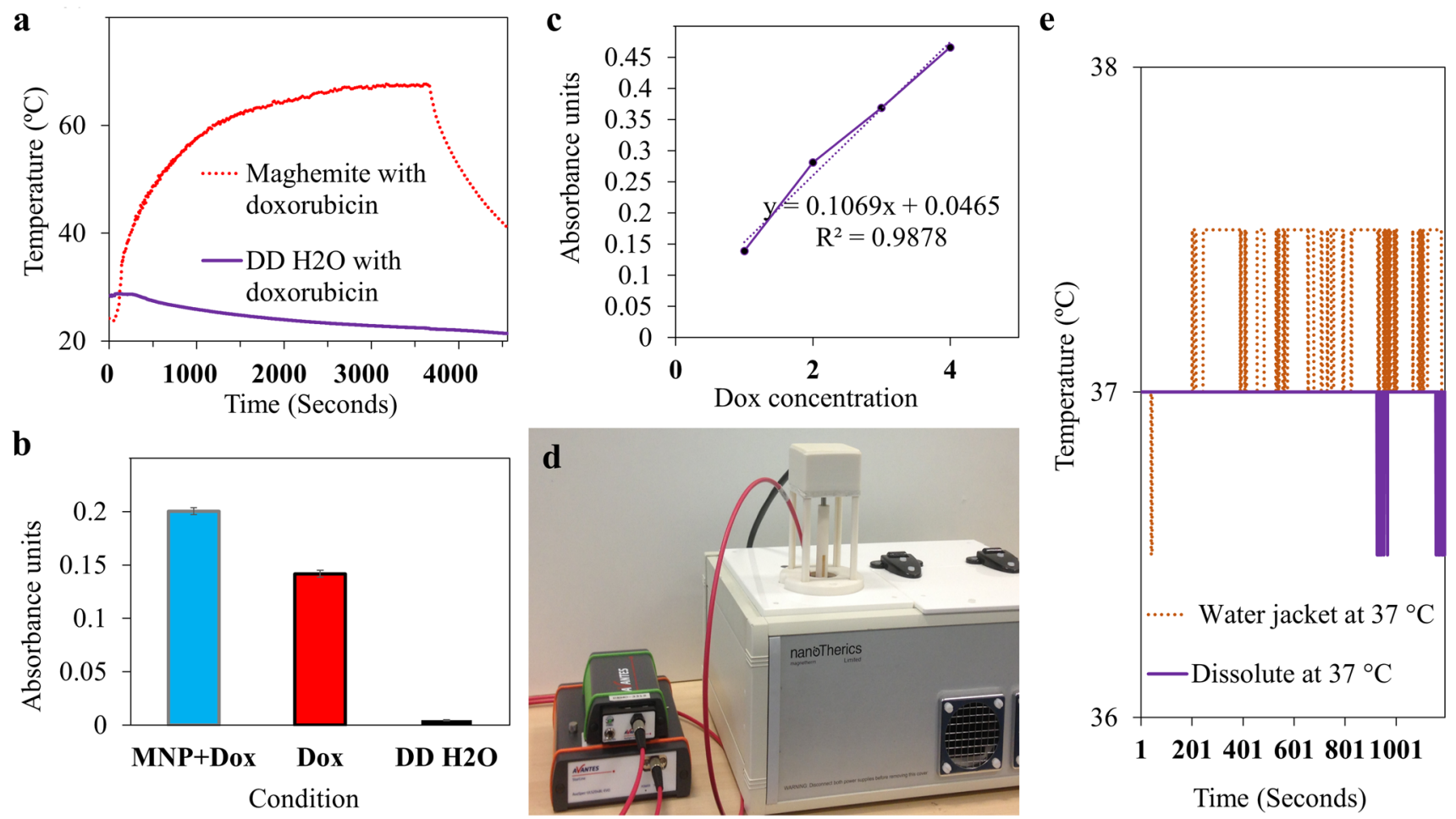

Fig. 4 a Real-time temperature changes in $50 \mathrm{~nm}$ sized maghemite nanoparticles $(2 \mathrm{ml}$ of $20 \mathrm{mg} / \mathrm{ml}$ ) dispersed in $100 \mu \mathrm{l}$ doxorubicin via a dialysis tube containing $25 \mathrm{ml}$ of double de-ionized water (DD $\mathrm{H}_{2} \mathrm{O}$ ); this was recorded using the AMF-mediated drug-release analysis setup. A fibre-optic temperature sensor was used for real-time temperature measurement and the sample-holding shaft was set to 20 rotations per minute. b Dox release measurement after $1 \mathrm{~h}$ of exposure to AMF at strength $16 \mathrm{mT}$ and frequency $107.7 \mathrm{kHz}$ followed by cooling for $15 \mathrm{~min}$ by maintaining the stirring rotation time at $20 \mathrm{rpm}$ - the real-time temperature measurement was recorded using an Osensa optical sensor. c Calibration curve for varying concentrations of doxorubicin. d Camera view of the experimental setup used for testing the AMF-mediated drug-release experiment: connected to a light source, a real-time UV-Vis absorption spectroscopy probe (compatible for AMF), and a spectrophotometer interface. e Temperature measurements for the water jacket without the dissolute and with the dissolute enclosed within the water jacket used in the drug-release testing setup. DD $\mathrm{H}_{2} \mathrm{O}$ only and MNP only with and without AMF exposure were used as controls

release should involve a high-power RF-AC magnetic field system with a coil, whose diameter can accommodate 1-5 $\mathrm{ml}$ of MNP-drug conjugate. The MNP-drug conjugate enclosed within a dialysis bag should be surrounded by release medium with volume at least 10 times that of the sample. The molecular weight cutoff of the dialysis membrane has to be high but should be able to withhold the MNP-drug conjugate, as the drug-release kinetics are limited by the permeability of the surface-functionalized Nano carriers. Use of a non-metallic temperature probe, a water jacket for maintaining physiological temperature, and drug-release media circulation to mimic blood circulation, in addition to caution for eddy current mediated nonspecific heating of the sample, was advised. Furthermore, they added that monitoring the drug-release profile continuously, while keeping the AMF ON will be beneficial for post experiment data analysis, as then the data will be derived from aliquots at specific intervals [21]. Moreover, all of the aforementioned points were incorporated into 
the design of the AMF-mediated drug-release system discussed in this study.

\subsection{Double-stacked planar coil system design}

The numerical calculations (as plotted in Fig. 5a-f) for the 2-layers stacked coil with 14 turns, result in an estimate for absolute flux density of $|B|=51 \mathrm{mT}$ and for RMS flux density of $B_{\mathrm{rms}}=36 \mathrm{mT}$, at coil current of $56 I_{\mathrm{rms}}$. This design demonstrates a potential of achieving higher flux densities with a relatively low amount of current through the coil and a reduction of heat generated by Joule's heating while current flows in the coil, when compared to the commonly used water-cooled induction coils (solenoid and planar). The highly intense and homogeneous flux line patterns at the centre of the coil (at the sample position) suggest that this is an ideal coil design for calorimetry experiments. The numerical simulations of $2 \times 16$ turn and $2 \times 15$ turn coils did provide better flux density, homogeneity, and intensity in the centre of the coil, as compared to those of a coil with 14 turns. Calculations based on $2 \times 15$ coils yielded an absolute flux density of $|B|=54.3 \mathrm{mT}$ and an RMS flux density of $B_{\text {rms }}=38.4 \mathrm{mT}$; and calculations based on $2 \times 16$ coils yielded an absolute flux density of $|B|=57.9 \mathrm{mT}$ and an RMS flux density of $B_{\mathrm{rms}}=46.3 \mathrm{mT}$; all these values were obtained for a coil current of $56 I_{\text {rms }}$.

However, because of fabrication issues, we used a $2 \times 14$ turn coil design (as shown in Fig. 6a-c) without compromising any measurements. A solenoid coil has usually been considered the appropriate choice in commercial instruments marketed for this purpose [22-25]. Hence, this high field-strength module was compared with a standard solenoid coil - the magneTherm system with dimensions $\mathrm{D}_{\mathrm{t}}=$ $4.06 \mathrm{~mm}, \mathrm{~d}_{\mathrm{t}}=2.68 \mathrm{~mm}, N=17, \mathrm{l}=2, S_{\mathrm{N}}=1.04 \mathrm{~mm}, S_{1}=$ $2.08 \mathrm{~mm}, d_{\mathrm{c}}=51.66 \mathrm{~mm}, D_{\mathrm{c}}=73.62 \mathrm{~mm}$, and $H=49.44$. This solenoid coil, however, produced a RMS B flux density of about $16.71 \mathrm{mT}$ at $56 \mathrm{I}_{\mathrm{rms}}$ coil current. The stacked planar coil did heat the MNP sample much more efficiently than
Fig. 5 a Simulated contour plots for the double-stacked planar coil with 14 turns in each stack for: the $x y$ plane absolute flux density $\left(\left|B_{x y}\right|\right)$, b the zy plane absolute flux density $\left(\left|B_{\text {zy }}\right|\right)$, and c the absolute flux density in the $x z$ plane of the coil applied with $I_{\text {rms }}=56 \mathrm{~A},|B|=51 \mathrm{mT}$. d The xy plane RMS flux density $\left(B_{\mathrm{xy}}\right)$, e the zy plane RMS flux density $\left(B_{z y}\right)$, and $\mathbf{f}$ the RMS flux density in the $x z$ plane for the coil applied with $I_{\mathrm{rms}}=$ $56 \mathrm{~A}, B_{\mathrm{rms}}=36 \mathrm{mT}$
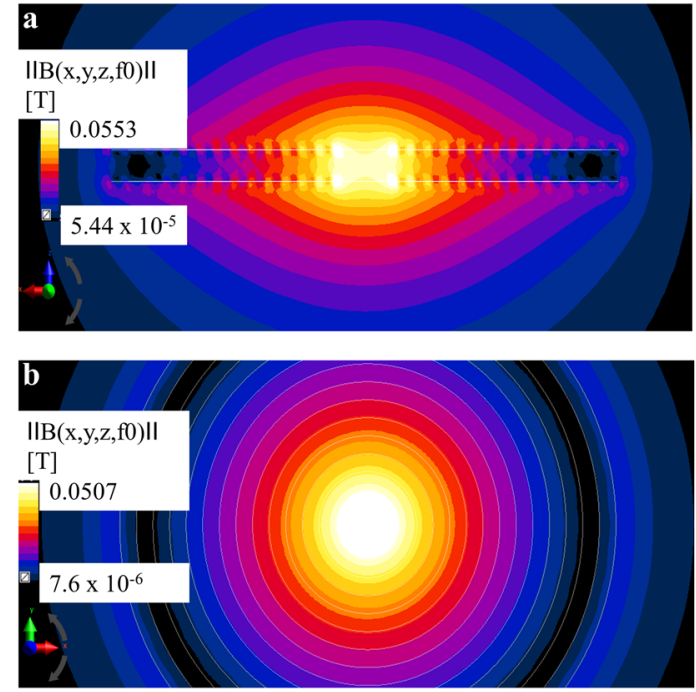

c
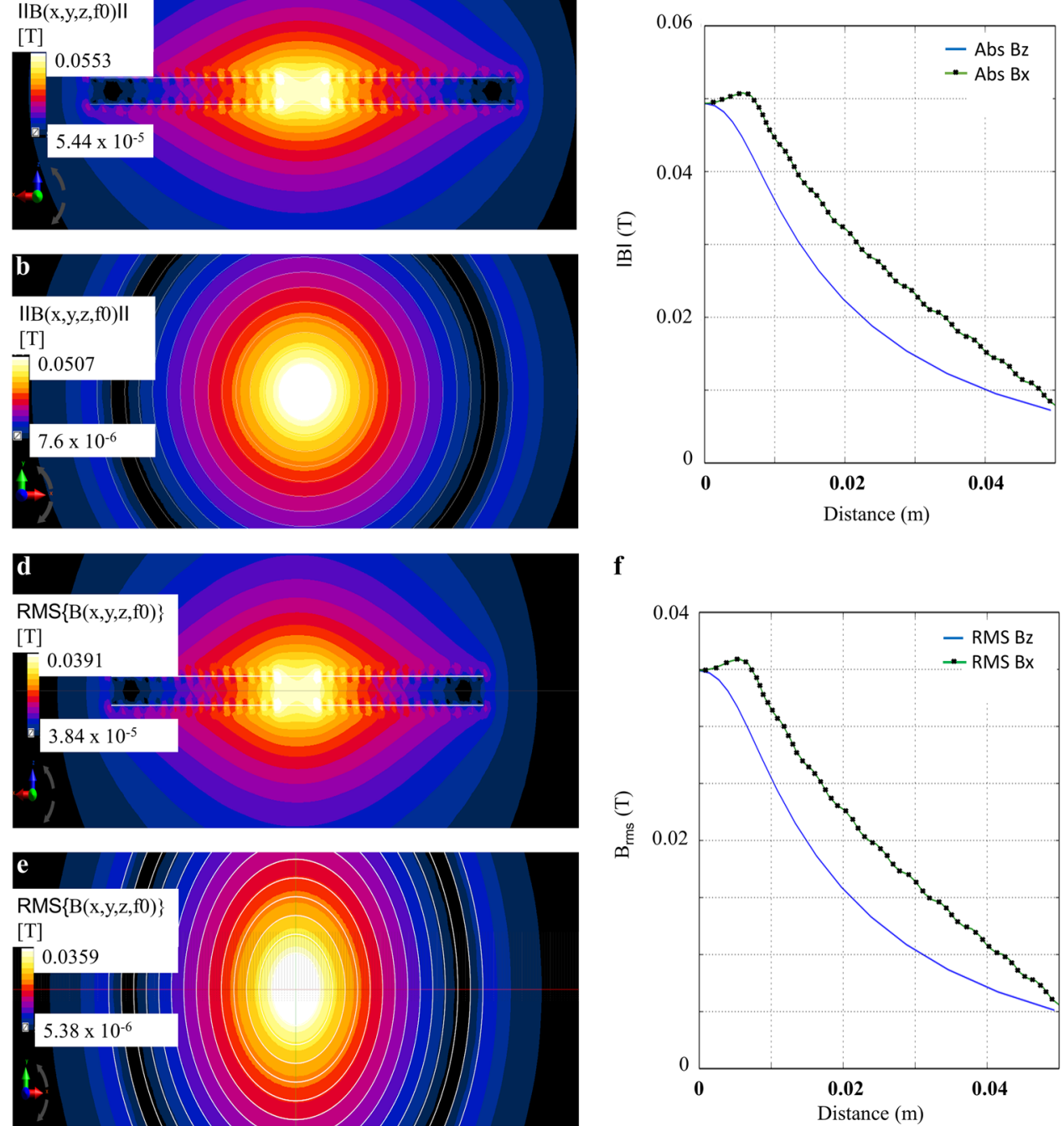
Fig. 6 a Fabricated high flux density coil setup: the stacked 2-layer planar coil; $\mathbf{b}$ the coil mount, to hold the stacked 2-layer planar coil, using a sample aperture; $\mathbf{c}$ and the Set up mounted externally onto the magneTherm ${ }^{\mathrm{TM}}$ system. d Change of temperature over time graph for a $5 \mathrm{mg}$ / $\mathrm{ml}$ aqueous suspension of DMSA stabilized $10.3 \mathrm{~nm}$ sized magnetite nanoparticles. A calorimetric experiment comparing double-stacked planar coil with a commercial setup using a simple solenoid at $56 I_{\mathrm{rms}}$ coil current. DD $\mathrm{H}_{2} \mathrm{O}$ only and $\mathrm{MNP}$ only with and without AMF exposure were used as controls
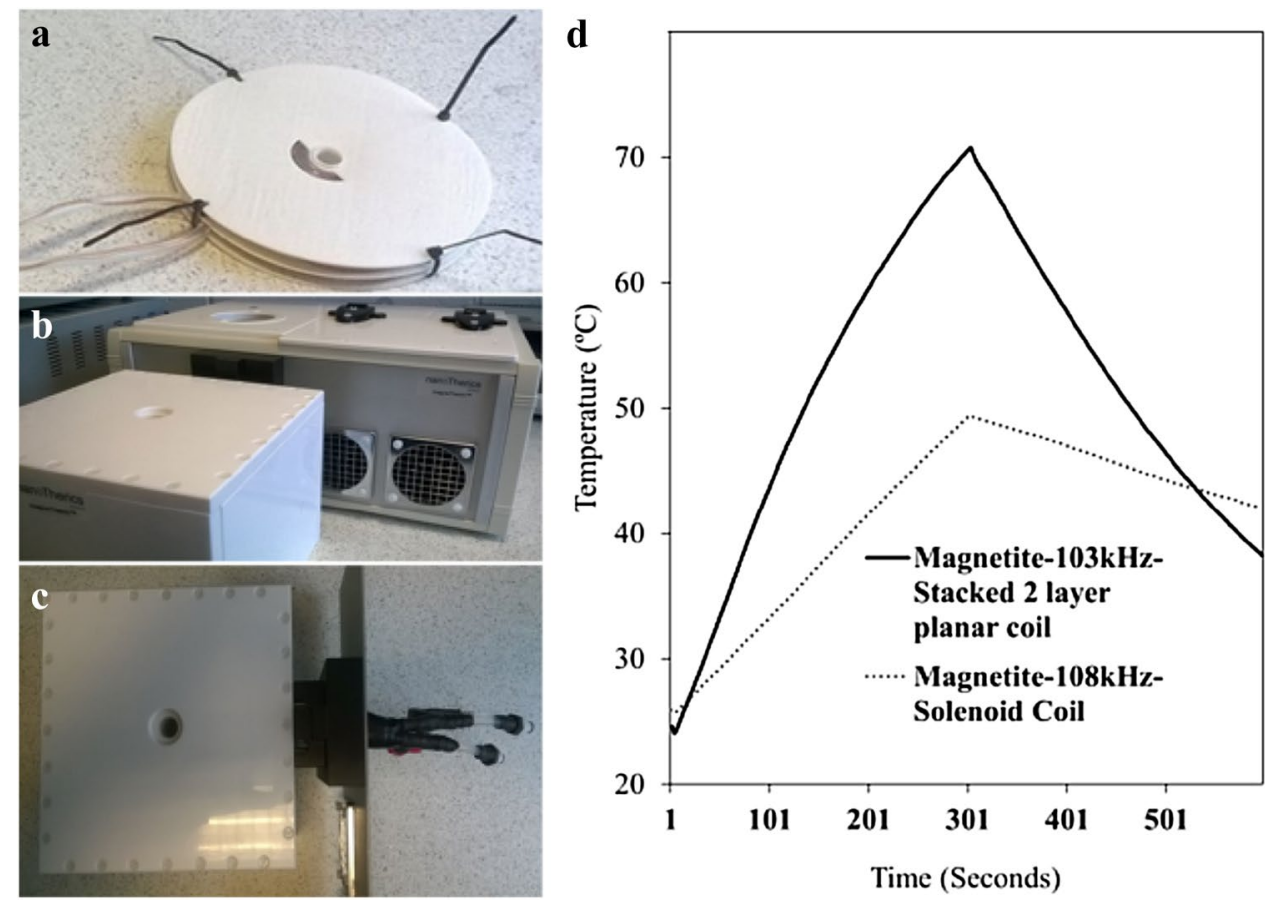

did the solenoid coil-as shown in Fig. 6d. The HFS coil design discussed here can be used for calorimetry as the centre of the coil can accommodate sample sizes up to $2 \mathrm{ml}$. Though the design does not include an adiabatic shield [26, 27], either vacuum layer or foam insulation can be included between the dielectric wall and the sample tube if the sample tube size is reduced to accommodate $1 \mathrm{ml}$ from the $2 \mathrm{ml}$ used in the study. However, it is evident from Figs. 5 and $6 \mathrm{~d}$, that this design provides enhanced effects in terms of incident flux intensity, homogeneity and flux density for the same coil current, when compared with a solenoid coil (with wide sample aperture) [10]. These parameters are the reason for observing increased heat dissipation in the calorimetry comparison results. In addition to induction coils, magnetic core-mediated heating is also plausible for MNP calorimetry experiments [28]. It is also possible to use a flux concentrator to influence the field parameters [29].

\section{Conclusion}

The measuring techniques and instrumentation developed here, demonstrated a cost-effective, accurate means of performing non-contact temperature measurements on in vitro samples. Likewise, the proposed drug-release analysis setup will allow researchers to perform and replicate their AMF-mediated drug-release experiments with accuracy and repeatability. We have also shown that the watercooled stacked planar coil system may be an efficient tool for performing MNP-calorimetric experiments. The aforementioned results infer that the tools and instrumentation proposed in this article has the potential to allow standardization of a variety of AMF-mediated MNP experiments with efficiency and repeatability.

Author contributions MS conceived the idea, designed and fabricated all the instruments described in the above article, MS and AM performed the evaluation experiments. MS, AM and JD performed data analysis. MS Wrote the manuscript. AKM and JD edited the manuscript.

\section{Compliance with ethical standards}

Conflict of interest The research was funded by nanoTherics Ltd. nanoTherics is involved with commercialisation of live cell - alternating magnetic field (LC-AMF) setup, Drug-Release Analysis System and High Flux Density module. MS worked as a research scientist in bioengineering for nanoTherics, and JD is a consultant for nanoTherics. Currently, MS is funded by the EPSRC and Imperial College London (EP/N509486/1: 1,979,819). AM and AKM are academic researchers and are unrelated to nanoTherics.

Open Access This article is distributed under the terms of the Creative Commons Attribution 4.0 International License (http://creativeco mmons.org/licenses/by/4.0/), which permits unrestricted use, distribution, and reproduction in any medium, provided you give appropriate credit to the original author(s) and the source, provide a link to the Creative Commons license, and indicate if changes were made. 


\section{References}

1. B. Kozissnik, A.C. Bohorquez, J. Dobson, C. Rinaldi, Magnetic fluid hyperthermia: advances, challenges and opportunity. Int. J. Hyperth. 29, 706-714 (2013)

2. M.A. Khan, C. Allemand, T.W. Eagar, Noncontact temperature measurement. II. Least squares based techniques. Rev. Sci. Instrum. 62, 403-409 (1991)

3. A. Skumiel, T. Hornowski, A. Józefczak, M. Koralewski, B. Leszczyński, Uses and limitation of different thermometers for measuring heating efficiency of magnetic fluids. Appl. Therm. Eng. 100, 1308-1318 (2016)

4. L.C. Rose et al., On-demand, magnetic hyperthermia-triggered drug delivery: optimisation for the GI tract. J. Mater. Chem. B. 4, 1704-1711 (2016)

5. T.M. Elkhova et al., The expeimental setup for measuring of thermal parameters of magnetic fluids in AC magnetic field. Solid. State. Phenom. 215, 454-458 (2014)

6. H. Rahn, S. Schenk, H. Engler, S. Odenbach, Tissue model for the study of heat transition during magnetic heating treatment. IEEE Trans. Magn. 49, 244-249 (2013)

7. H.F. Rodrigues et al., Real-time infrared thermography detection of magnetic nanoparticle hyperthermia in a murine model under a non-uniform field configuration. Int. J. Hyperth. 29, 752-767 (2013)

8. H.L. Rodríguez et al., Studies of ferrite based magnetic nanoparticle uptake and magnetocytolysis effects on model cell cultures. Nanotech. 2, 321-324 (2009)

9. M. Subramanian, A. Miaskowski, G. Pearce, J. Dobson, A coil system for real-time magnetic fluid hyperthermia microscopy studies. Int. J. Hyperth. 32, 112-120 (2016)

10. M. Subramanian et al., A Pilot study into the use of FDG mNP as an alternative approach in neuroblastoma cell hyperthermia. IEEE Trans. Nanobiosci. 15, 517 (2016)

11. S.L. Fossheim, K.A. Il'yasov, J. Hennig, A. Bjørnerud, Thermosensitive paramagnetic liposomes for temperature control during MR imaging-guided hyperthermia: in vitro feasibility studies. Acad. Radiol. 7(12), 1107-1115 (2000)

12. N.S. Satarkar, J.Z. Hilt, Magnetic hydrogel nanocomposites for remote controlled pulsatile drug release. J. Control. Release. 130, 246-251 (2008)

13. A. Teleki, F.L. Haufe, A.M. Hirt, S.E. Pratsinis, G.A. Sotiriou, Highly scalable production of uniformly-coated superparamagnetic nanoparticles for triggered drug release from alginate hydrogels. RSC Adv. 6, 21503-21510 (2016)

14. X. Ding et al., Sol-gel system functionalized magnetic nanocubes as remote controlled drug carriers for cooperative tumor-targeted therapy. Mater. Lett. 175, 236-240 (2016)

15. S. Brulé et al., Doxorubicin release triggered by alginate embedded magnetic nanoheaters: a combined therapy. Adv. Mater. 23, 787-790 (2011)

16. A. Hervault, A.E. Dunn, M. Lim, C. Boyer, D. Mott, S. Maenosono, N.T. Thanh, Doxorubicin loaded dual $\mathrm{pH}$-and thermo-responsive magnetic nanocarrier for combined magnetic hyperthermia and targeted controlled drug delivery applications. Nanoscale 8(24), 12152-12161 (2016)

17. S. D'Souza, A review of in vitro drug release test methods for nano-sized dosage forms. Adv. Pharm. 1-12 (2014)

18. R.R. Wildeboer, P. Southern, Q.A. Pankhurst, On the reliable measurement of specific absorption rates and intrinsic loss parameters in magnetic hyperthermia materials. J. Phys. D Appl. Phys. 47, 495003 (2014)

19. L.R. Silva, M.D. Stuart, Fluke, Corporation, Method of combined use of infrared camera, non-contact infrared sensor, or contact temperature sensor with insulation resistance tester for automatic temperature normalization. U.S. Patent 20,160,131,607 (2016)

20. F. Benyettou, O. Flores, J. Alonso, F. Ravaux, R. Rezgui, M. Jouiad, S.I. Nehme, R.K. Parsapur, J.C. Olsen, P. Selvam, A. Trabolsi, Mesoporous $\gamma$-iron oxide nanoparticles for magnetically triggered release of doxorubicin and hyperthermia treatment. Chem. A Eur. J. 22(47), 17020-17028 (2016)

21. D. Mertz, O. Sandre, S. Begin-Colin, Drug releasing nanoplatforms activated by alternating magnetic fields. Biochimica et Biophys. Acta (BBA) Gen. Subj. 1861(6), 1617-1641 (2017)

22. B.I. Macías-Martínez, D.A. Cortés-Hernández, A. Zugasti-Cruz, B.R. Cruz-Ortíz, E.M. Múzquiz-Ramos, Heating ability and hemolysis test of magnetite nanoparticles obtained by a simple coprecipitation method. J. Appl. Res. Technol. 14, 239-244 (2016)

23. N. Iacob et al., Effects of magnetic dipolar interactions on the specific time constant in superparamagnetic nanoparticle systems. J. Phys. D Appl. Phys. 49, 295001 (2016)

24. M.P. Calatayud, L. Asin, A. Tres, G.F. Goya, M.R. Ibarra, Cell bystander effect induced by radiofrequency electromagnetic fields and magnetic nanoparticles. Curr. Nanosci. 12, 372-377 (2016)

25. P.I. Soares et al., Iron oxide nanoparticles stabilized with a bilayer of oleic acid for magnetic hyperthermia and MRI applications. Appl. Surf. Sci. 383, 240-247 (2016)

26. E. Natividad, M. Castro, A. Mediano, Adiabatic magnetothermia makes possible the study of the temperature dependence of the heat dissipated by magnetic nanoparticles under alternating magnetic fields. Appl. Phys. Lett. 98(24), 243119 (2011)

27. E. Natividad, M. Castro, A. Mediano, Adiabatic vs. non-adiabatic determination of specific absorption rate of ferrofluids. J. Magn. Magn. Mater. 321(10), 1497-1500 (2009)

28. C.C. Tai, C.C. Chen, The design of a half-bridge series-resonant type heating system for magnetic nanoparticle thermotherapy. PIERS Online 4(2), 276-280 (2008)

29. R. Ivkov, S.J. DeNardo, W. Daum, A.R. Foreman, R.C. Goldstein, V.S. Nemkov, G.L. DeNardo, Application of high amplitude alternating magnetic fields for heat induction of nanoparticles localized in cancer. Clin. Cancer Res. 11(19), 7093s-7103s (2005)

Publisher's Note Springer Nature remains neutral with regard to jurisdictional claims in published maps and institutional affiliations. 